\title{
Active planar optical waveguide made from luminescent silicon nanocrystals
}

\author{
J. Valenta \\ Department of Chemical Physics and Optics, Faculty of Mathematics and Physics, Charles University, \\ Ke Karlovu 3, CZ-121 16 Prague 2, Czech Republic \\ I. Pelant ${ }^{\mathrm{a})}$ and K. Luterová \\ Institute of Physics, Academy of Sciences of the Czech Republic, Cukrovarnická 10, CZ-162 53 Prague 6, \\ Czech Republic \\ R. Tomasiunas \\ Institute of Materials Science and Applied Research, Vilnius University, 2054 Vilnius, Lithuania \\ S. Cheylan and R. G. Elliman \\ Electronic Materials Engineering Department, Research School of Physical Sciences and Engineering, \\ Australian National University, Canberra, ACT 0200, Australia \\ J. Linnros \\ Department of Microelectronics and Information Technology, Royal Institute of Technology, Electrum 229, \\ S-164 40 Kista-Stockholm, Sweden

\section{B. Hönerlage} \\ IPCMS, Groupe d'Optique Nonlinéaire et d'Optoéléctronique, UMR 7504 CNRS-ULP, 23, rue du Loess, \\ 67037 Strasbourg Cedex, France
}

(Received 2 July 2002; accepted 17 December 2002)

\begin{abstract}
We show experimentally that a layer of silicon nanocrystals, prepared by the Si-ion implantation (with the energy of $400 \mathrm{keV}$ ) into a synthetic silica slab and exhibiting room-temperature red photoluminescence, can serve simultaneously as a single-mode planar optical waveguide. The waveguide is shown to self-select guided transverse electric and transverse magnetic modes from the broad photoluminescence emission of the nanocrystals resulting in a substantially narrower emission spectrum for these modes. We further report on an investigation of optical gain in a sample implanted to a dose of $4 \times 10^{17} \mathrm{~cm}^{-2}$. Despite the occurrence of strong waveguiding, results of the variable stripe length method turned out not to be able to give unambiguous evidence for optical gain. (C) 2003 American Institute of Physics. [DOI: 10.1063/1.1544433]
\end{abstract}

The ongoing interest in Si-based optoelectronic devices has recently been further stimulated by reports of optical gain and stimulated emission in layers containing silicon nanocrystals (Si-NCs). ${ }^{1-5}$ This research is motivated by the technological prospect of making a silicon-based laser and by questions of fundamental importance, such as whether or not it is possible to overcome the constraints imposed by an indirect band gap by exploiting the properties of nanoscale material structures. Studies have largely concentrated on the properties of ensembles of Si-NCs embedded in a $\mathrm{SiO}_{2}$ matrix. For example, waveguiding structures fabricated from erbium-doped $\mathrm{Si}$-NCs embedded in a $\mathrm{SiO}_{2}$ matrix have recently been shown to exhibit amplification of an externally coupled signal near $1.5 \mu \mathrm{m}$ under optical pumping. ${ }^{6}$ The prospect of exploiting the waveguiding properties of nccontaining layers is particularly interesting since such guiding could enhance optical gain in such layers by eliminating losses as well as provide a useful basis for the fabrication of other Si-based optoelectronic devices and structures. In this letter the waveguiding properties of undoped Si-NCs containing layers are examined in detail. We further discuss the

a)Electronic mail: pelant@fzu.cz application of the variable stripe length (VSL) $\operatorname{method}^{7}$ for investigating the occurrence of gain near $750 \mathrm{~nm}$ in our waveguide structure.

Samples used in this study were prepared by implantation of $\mathrm{Si}^{+}$ions into synthetic silica slabs (Infrasil) with dimensions of $10 \times 5 \times 1 \mathrm{~mm}^{3}$ and with optically polished faces. The implantation energy was $400 \mathrm{keV}$ and ion doses were varied in the range $1-6 \times 10^{17} \mathrm{~cm}^{-2}$. This resulted in slightly skewed implant distributions with a mean project range of $\sim 630 \mathrm{~nm}$ and peak excess concentration of up to 26 at. \% Si. The implanted samples were submitted to a postimplantation anneal at $1100{ }^{\circ} \mathrm{C}$ in a $\mathrm{N}_{2}$ ambient for $1 \mathrm{~h}$ to form Si-NCs. To enhance the photoluminescence (PL) intensity from NCs owing to hydrogen pasivation, an additional $1 \mathrm{~h}$ anneal $^{8}$ was performed in a forming gas $\left(95 \% \mathrm{~N}_{2} / 5 \% \mathrm{H}_{2}\right)$ ambient at $500{ }^{\circ} \mathrm{C}$. The presence of Si-NCs was evidenced by Raman spectra that exhibited typical shift and line broadening consistent with a mean $\mathrm{NC} \operatorname{size}^{9}$ of $\sim 3 \mathrm{~nm}$. Based on the implanted Si distribution ${ }^{10}$ an estimated peak density of NCs varies in the range $1-2 \times 10^{19} \mathrm{~cm}^{-3}$. PL was studied using two independent setups. The first one (Royal Institute of Technology, Stockholm) used a continuous wave (cw) $\mathrm{He}-\mathrm{Cd}$ laser $(325 \mathrm{~nm})$ as the excitation source and an imaging spectrograph with a CCD camera for detection. In the 


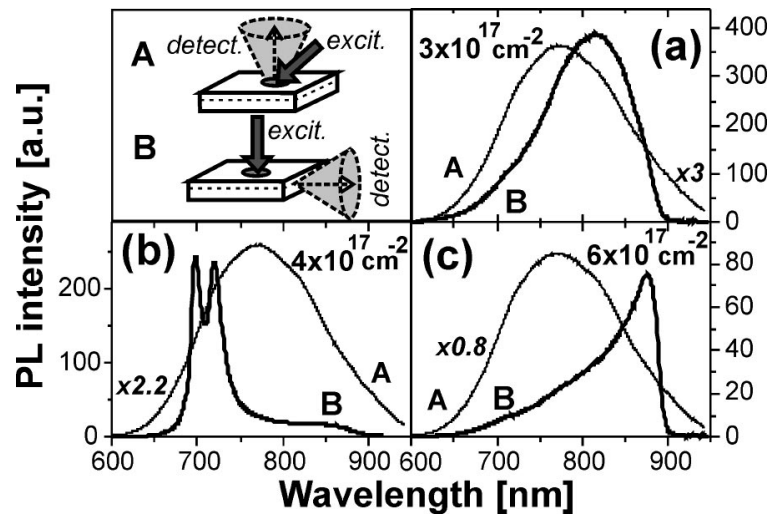

FIG. 1. Unpolarized PL spectra at 295 K. Curves A correspond to PL emanating from the surface of the samples (perpendicular to the $\mathrm{Si}-\mathrm{NC}$ layer) while the curves B represent PL collected from the facet of the samples (in the plane containing $\mathrm{Si}-\mathrm{NCs}$ ) (see inset). Emission spectra of the sample $1 \times 10^{17} \mathrm{~cm}^{-2}$ (not shown) are similar to those exhibited by the sample 3 $\times 10^{17} \mathrm{~cm}^{-2}$ and displayed in panel (a). (b) and (c) show results for samples implanted to doses of $4 \times 10^{17}$ and $6 \times 10^{17} \mathrm{~cm}^{-2}$, respectively. (Excitation: cw $325 \mathrm{~nm}$, detection by the CCD camera).

second PL setup (Institut de Physique et Chimie des Matériaux de Strasbourg), PL was excited with a pulsed $\mathrm{XeCl}$ excimer laser (308 $\mathrm{nm}$, pulse duration $\sim 18 \mathrm{~ns}$ ) and analyzed in a scanning monochromator equipped with a GaAs photomultiplier and a boxcar integrator. This apparatus was also used to investigate optical gain via the VSL method in the temperature range from 8 to $292 \mathrm{~K}$ using a gas-flow optical cryostat.

The waveguiding properties of some of the investigated layers manifest themselves clearly as striking differences in PL spectra detected from the surface or from the facet of the sample, as demonstrated in Fig. 1 for samples with different implantation fluences. While for fluences of $1 \times 10^{17} \mathrm{~cm}^{-2}$ (not shown) and $3 \times 10^{17} \mathrm{~cm}^{-2}$ the emission spectra for both directions of detection correspond to the broad, structureless emission characteristic of nanometer-sized $\mathrm{Si}-\mathrm{NCs},{ }^{8,11,12}$ the spectrum collected from the facet of the $4 \times 10^{17} \mathrm{~cm}^{-2}$ sample differs significantly. It is much narrower and exhibits two distinct peaks. (The spectral position of the peaks is slightly different for different batches of samples [compare Figs. 1(b) and 2(a)] but the general shape is reproducible.) Similar behavior is evident also in the sample implanted to a dose $6 \times 10^{17} \mathrm{~cm}^{-2}$.

We interpret this effect as being due to waveguiding of the red PL emission in the luminescent layer composed of Si-NCs. Due to the relatively high implantation energy, the resulting layer composed of $\mathrm{Si}-\mathrm{NCs}$ is buried in the $\mathrm{SiO}_{2}$ matrix providing the refractive index contrast indispensable for waveguiding: It possesses an increased refractive index (up to $n_{1} \approx 1.73$ ) compared with the surrounding matrix $\left(n_{2} \approx 1.455\right)$ and is relatively thin (thickness $t$ is about 310 $\mathrm{nm}) .{ }^{10}$ In the first approximation we can model the layer as a symmetric planar waveguide (with a rectangular refractive index profile). Then, the condition for single mode guiding, ${ }^{13}$ i.e., $0<\sqrt{\left(n_{1}^{2}-n_{2}^{2}\right)}(\omega / c) t<\pi$, is fulfilled for wavelengths $\lambda=2 \pi c / \omega$ longer than $\sim 590 \mathrm{~nm}$. Indeed, investigation of the PL polarization revealed that the two peaks are clearly polarization resolved TE and TM modes [Fig. 2(a)].

It is worth stressing that in these experiments the PL

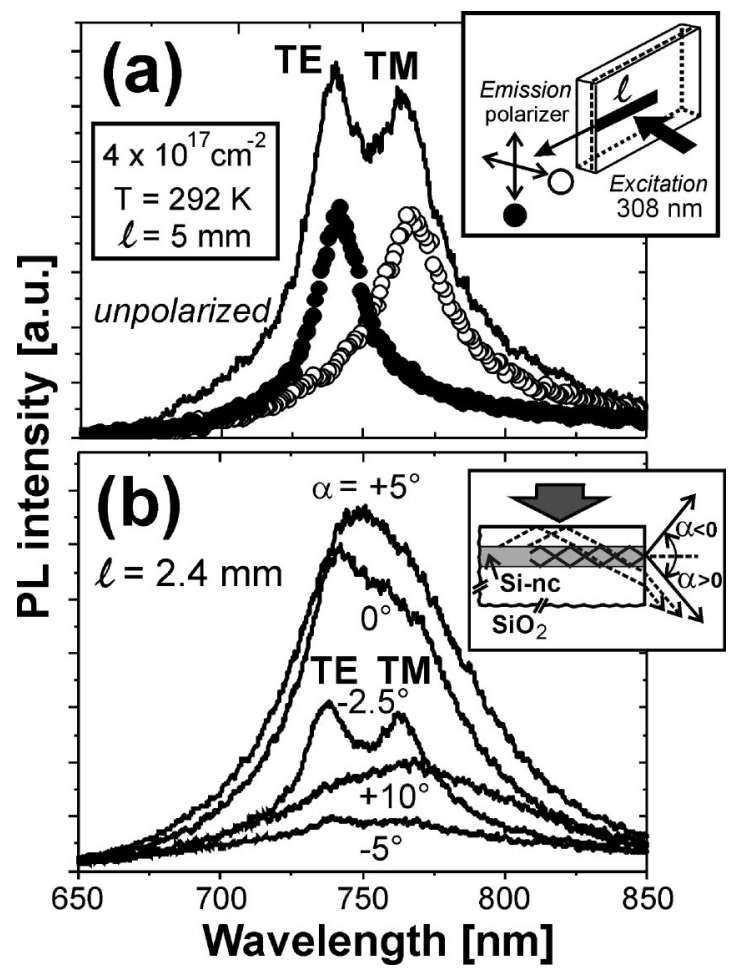

FIG. 2. (a) Room-temperature emission spectra collected from the facet of the sample implanted to a dose of $4 \times 10^{17} \mathrm{~cm}^{-2}$ under $600 \mathrm{~kW} / \mathrm{cm}^{2}$ excitation with a $\mathrm{XeCl}$ laser (see inset). The solid line represents unpolarized PL, solid symbols show spectrum obtained by inserting a polarizer between the sample and the monochromator ( $\mathbf{E}$ is perpendicular to the plane defined by the excitation and emission beams, TE mode). Open symbols denote PL spectrum when the polarizer is turned by $90^{\circ}$ (TM mode). (b) Unpolarized PL spectra measured under various angles $\alpha$ with respect to the normal to the output facet. The emission beam remains in the plane set by the normal and by the excitation beam as indicated in the inset. Inset also shows a scheme explaining the observed asymmetry of emission intensity with respect to $\alpha$. Solid (dashed) lines represent guided (unguided) PL.

generated by the layer itself serves as the test beam probing the waveguide properties, without the necessity of any external coupler. The waveguide obviously self-selects the discrete guided modes from the broad PL spectrum. Such behavior, also recently reported in Ref. 2, is quite interesting and possibly results from a "microcavity" effect in which the NC emission is modified by the available optical density-of-states. ${ }^{14}$ This, coupled with the modified refractive index profile resulting from the different implant conditions, may also explain the observed spectral shift of the modes compared to the emission from the surface [a blueshift for a dose of $4 \times 10^{17} \mathrm{~cm}^{-2}$ - see Fig. 1(b) —and a red one for $6 \times 10^{17} \mathrm{~cm}^{-2}$, Fig. 1(c)].

The waveguide properties together with the corresponding strong directionality of the spectral features are further demonstrated in Fig. 2(b). By collecting PL under various angles $\alpha$ with respect to the layer, the structure of guided emission is shown to be a sensitive function of detection angle - the clearly resolved peaks corresponding to TE and TM modes are observable over a narrow range of $\alpha$ only. When the guided PL is detected at angles greater than $\alpha$ $\approx \pm 5^{\circ}$, the spectrum becomes broad and structureless, obviously due to an increasing contribution of unguided (leakage) PL. Figure 2(b) also highlights another surprising aspect of the emission-the overall structure is not symmetric with respect to $\alpha=0^{\circ}$ and the total PL intensity has a maximum at license or copyright; see http://apl.aip.org/about/rights_and_permissions 


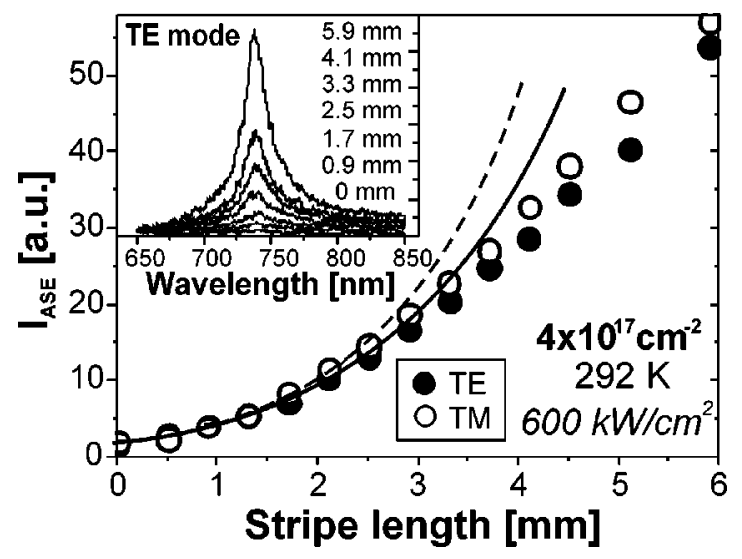

FIG. 3. Example of VSL investigation of optical gain $(T=292 \mathrm{~K}$, excitation: $\mathrm{XeCl}$ laser $308 \mathrm{~nm}$ ) in the sample implanted to a dose of 4 $\times 10^{17} \mathrm{~cm}^{-2}$. The experimental geometry is sketched in the inset of Fig. 2(a). Stripe width was about $0.1 \mathrm{~mm}$; inset shows selected emission spectra for various stripe lengths (a parameter next to the curves).

$\alpha=+5^{\circ}$ while it is mostly suppressed at $\alpha=-5^{\circ}$. This output asymmetry can be qualitatively understood by taking into account the asymmetric location of the active layer with respect to the surrounding $\mathrm{SiO}_{2}$ matrix, close to the sample surface, as shown in the inset of Fig. 2(b). The total internal reflection of unguided PL on the sample surface entails enhancement of the output intensity at $\alpha>0^{\circ}$. Possible rounding of the sample edges (not perfectly perpendicular) could also deflect the outcoming light to the $\alpha>0^{\circ}$ direction.

To assess the potential influence of waveguiding on the occurrence of optical gain in a slab Si-NC waveguide, we applied the VSL technique to the sample implanted to a dose of $4 \times 10^{17} \mathrm{~cm}^{-2}$. Our previous measurements using $\mathrm{cw}$ pumping at $325 \mathrm{~nm}$ revealed no gain. ${ }^{15}$ Here we extend those measurements by using a $308 \mathrm{~nm}$ pulsed high density excitation, performed moreover at various temperatures between 8 and $295 \mathrm{~K}$. The principle of the VSL method is recalled in the inset of Fig. 2(a). A plot of the output guided PL amplified spontaneous emission $I_{\mathrm{ASE}}$ against the excited stripe length $l$ can be described as $I_{\mathrm{ASE}^{\propto}\left(I_{s p} / g\right) \cdot[\exp (g l)-1]}$ where $I_{s p}$ is spontaneous emission per unit length and $g$ is net optical gain (=total gain-loss). An initial exponential increase in intensity was indeed reproducibly found at wavelengths of the TE and TM modes (see Fig. 3). Fitting by the above equation (full and dotted lines) yields $g$ about 6 and $8 \mathrm{~cm}^{-1}$ for TE and TM modes, respectively (we point out that gain of $6 \mathrm{~cm}^{-1}$ at $720 \mathrm{~nm}$ has been reported recently also by Khriachtchev et al. ${ }^{2}$ under pumping with ns pulses). However, we stress that this simplistic interpretation of the VSL measurements requires some caution because the VSL method (originally developed for direct-gap semiconductors with gain of $10^{2}$ up to $\left.10^{4} \mathrm{~cm}^{-1}\right)^{7}$ is not ideally suited for investigating small gains. In particular, the initial faint exponential growth can result from several side effects (such as the finite numerical aperture of the collecting optics, a re- sidual signal background, total internal reflection at the output facet, etc.), not necessarily from true optical gain. Analysis of these effects will be published elsewhere. ${ }^{16}$ Indirect evidence for the lack of gain is also provided in the present analysis by the fact that neither the overall behavior of the curves shown in Fig. 3 nor the extracted values of $g$ vary significantly when the pump density is varied $\left(200-600 \mathrm{~kW} / \mathrm{cm}^{2}\right)$. Furthermore, no noticeable variations were found when the temperature was varied over a wide range $(8-292 \mathrm{~K})$. Clearly the problem needs further investigation.

In conclusion, we have presented experimental data demonstrating the waveguiding properties of a layer composed of Si nanocrystals embedded in a silica slab. Room temperature guided emission in the red spectral region, originating from the luminescent nanocrystals, was shown to contain polarization distinguished TE and TM peaks, the structure of which was a sensitive function of observation angle. However, the application of the VSL method, either with a cw $325 \mathrm{~nm}$ or a nanosecond $308 \mathrm{~nm}$ pumping, did not give conclusive evidence for optical gain in the waveguide.

This work was supported by Project Nos. A1010809 and AVOZ 1010914 of GAAVCR, Project 202/01/D030 of GACR, by NATO (PST.CLG.978100), and by the Royal Swedish Academy of Sciences. One of the authors (R.T.) appreciates the financial support from the Lithuanian State Foundation for Science and Studies. We would like to thank to Dr. P. Gilliot, Dr. O. Crégut, and Dr. V. Vorlícek for experimental assistance.

${ }^{1}$ L. Pavesi, L. Dal Negro, C. Mazzoleni, G. Franzo, and F. Priolo, Nature (London) 408, 440 (2000).

${ }^{2}$ L. Khriachtchev, M. Räsänen, S. Novikov, and J. Sinkkonen, Appl. Phys. Lett. 79, 1249 (2001).

${ }^{3}$ M. H. Nayfeh, S. Rao, N. Barry, J. Therrien, G. Belomoin, and A. Smith, Appl. Phys. Lett. 80, 121 (2002).

${ }^{4}$ M. Nayfeh, O. Akcakir, S. J. Therrien, Z. Yamani, N. Barry, W. Yu, and E. Gratton, Appl. Phys. Lett. 75, 4112 (1999).

${ }^{5}$ M. H. Nayfeh, N. Barry, J. Therrien, O. Akcakir, E. Gratton, and G. Belomoin, Appl. Phys. Lett. 78, 1131 (2001).

${ }^{6}$ H.-S. Han, S.-Y. Seo, and J. H. Shin, Appl. Phys. Lett. 79, 4568 (2001).

${ }^{7}$ K. L. Shaklee, J. Lumin. 7, 284 (1973).

${ }^{8}$ S. Cheylan and R. G. Elliman, Appl. Phys. Lett. 78, 1225 (2001).

${ }^{9}$ J. Zi, H. Büscher, C. Falter, W. Ludwig, K. Zhang, and X. Xie, Appl. Phys. Lett. 69, 200 (1996)

${ }^{10}$ R. G. Elliman, M. J. Lederer, and B. Luther-Davies, Appl. Phys. Lett. 80, 1325 (2002)

${ }^{11}$ S. Guha, J. Appl. Phys. 84, 5210 (1998).

${ }^{12}$ J. Linnros, N. Lalic, A. Galeckas, and V. Grivickas, J. Appl. Phys. 86, 6128 (1999).

${ }^{13}$ A. Yariv and P. Yeh, Optical Waves in Crystals (Wiley, New York, 1984).

${ }^{14}$ T. Gutbrod, M. Bayer, A. Forchel, P. A. Knipp, T. L. Reinecke, A. Tartakovskii, V. D. Kulakovskii, N. A. Gipius, and S. G. Tikhodeev, Phys. Rev. B 59, 2223 (1999).

${ }^{15}$ J. Valenta, I. Pelant, and J. Linnros, Appl. Phys. Lett. 81, 1396 (2002).

${ }^{16}$ J. Valenta, K. Luterova, R. Tomasiunas, K. Dohnalova, B. Hönerlage, and I. Pelant, Towards the First Silicon Laser, edited by L. Pavesi, S. Gaponenko, and L. Dal Negro, NATO Science Series (Kluwer, Dordrecht, 2003), to be published. 